\title{
MicroRNA-34a attenuates the proliferation, invasion and metastasis of gastric cancer cells via downregulation of MET
}

\author{
BIN WEI $^{1 *}$, QIAO YING HUANG ${ }^{2 *}$, SHUN RONG HUANG $^{1}$, WEI MAI ${ }^{1}$ and XIAO GANG ZHONG ${ }^{1}$ \\ ${ }^{1}$ Department of Gastroenterology and Peripheral Vascular Surgery, People's Hospital of Guangxi Zhuang Autonomous Region; \\ ${ }^{2}$ Department of Blood Transfusion, The First Affiliated Hospital, Guangxi Medical University, \\ Nanning, Guangxi 530000, P.R. China
}

Received August 25, 2014; Accepted May 1, 2015

DOI: $10.3892 / \mathrm{mmr} .2015 .4110$

\begin{abstract}
Proliferation, invasion and metastasis are key features of gastric cancer, contributing to high mortality rates in patients with gastric cancer worldwide. As a direct target of p53, the functions of microRNA (miR)-34a are important, but controversial, in the progression of gastric cancer. In the present study, the clinical importance of miR-34a in GC specimens $(n=40)$ were investigated and were confirmed in an independent cohort from The Cancer Genome Atlas (TCGA; $\mathrm{n}=352$ ). The prognostic value of miR-34a was analyzed using a Kaplan-Meier survival curve in the TCGA cohort, in combination with complete follow-up data $(n=157)$. The level of miR-34a was detected in the human gastric cancer cell line and normal gastric epithelial cell line. The effect of miR-34a on proliferation and invasion were evaluated using Cell Counting Kit 8, colony formation and cell invasion assays. The molecular basis of miR-34a was determined by bioinformatics prediction. The correlation between miR-34a and MET was assessed using reverse transcription-quantitative polymerase chain reaction and western blot analyses. The results indicated that miR-34a was downregulated in the gastric cancer tissues, compared with the normal gastric tissues $(\mathrm{P}<0.01)$. miR-34a was negatively correlated with the depth of invasion and lymph node metastasis of gastric cancer $(\mathrm{P}<0.01)$. In the TCGA cohort, the levels of miR-34a were lower in T3 and T4 tumor stages, compared with the level in the T1 stage, and low levels of miR-34a predicted significantly longer survival rates in patients with GC $(\mathrm{P}<0.05)$. miR-34a also attenuated the proliferation ability, and inhibited the colony formation and
\end{abstract}

Correspondence to: Dr Xiao Gang Zhong, Department of Gastroenterology and Peripheral Vascular Surgery, People's Hospital of Guangxi Zhuang Autonomous Region, 6 Taoyuan Road, Nanning, Guangxi 530000, P.R. China

E-mail: zhongxg_ph@126.com

*Contributed equally

Key words: gastric cancer, microRNA-34a, prognosis, proliferation, invasion/metastasis, MET cell invasion abilities of the cells $(\mathrm{P}<0.01)$. A negative correlation was observed between miR-34a and MET in gastric cancer $(\mathrm{P}<0.01 ; \mathrm{r}=-0.9526)$, and $>60 \%$ of cases exhibited consistent expression of miR-34a and MET in gastric cancer $(\mathrm{P}<0.01)$. In conclusion, miR-34a was associated with the clinicopathological features of gastric cancer and was a valuable predictor of patient prognosis. miR-34a acted as a tumor suppressor to inhibit gastric cancer proliferation and invasion via the downregulation of MET.

\section{Introduction}

Gastric cancer (GC) is one of the most prevalent types of malignancy worldwide and contributes to the second most common cause of cancer-associated mortality worldwide $(1,2)$. The pathogenesis of GC is a multistep process, involving single or multiple mutations of genes associated with cell proliferation, invasion and metastasis (3). The prognosis of patients with GC is poor, with a 5-year overall survival rate of $28 \%$ worldwide $(1,2)$. One of the key reasons for the poor prognosis of patients with GC is its character of invasion and metastasis, which has been observed to occur in $>60 \%$ of patients at the point of diagnosis (4-6). Therefore, it's important to understand the molecular basis of GC invasion and metastasis in order to develop novel therapeutic strategies for GC (7). The discovery of microRNAs (miRNAs) is a significant milestone in the investigation of cancer, including GC (8).

miRNAs are small, non-cording RNAs, 17-25 nucleotides in length, and are involved in the post-transcriptional regulation of hundreds of target genes. miRNA-protein complexes can bind to the mRNA of target genes, leading to degradation or translational inhibition of target genes, which controls a wide range of biological functions, including cell proliferation, differentiation and apoptosis (8-10). miRNAs have been a major focus in studies investigating cancer $(4,7,11,12)$. Increasing evidence indicates that miRNAs have paradoxical functions and act in a cell context-dependent manner $(13,14)$.

The has-miR-34 family is located in Ch1p36, a region that is frequently deleted in tumors (15). It has been reported that miR-34b/c is decreased in human non-small cell lung cancer cell lines, however, miR-34a cannot simply be defined as a tumor suppressor or oncogene, as paradoxical roles of miR-34a have been observed in several types of cancer (16). In 
human glioma, miR-34a reduces the expression of Notch and acts as a tumor suppressor (17). The same effect of miR-34a has been observed in human hepatocellular carcinoma and GC $(18,19)$. However miR-34a is upregulated in hepatocellular carcinoma and is associated with hepatocarcinogenesis (20). A GC miRNA profile also demonstrates that miR-34a is overexpressed in GC tissues, compared with normal gastric tissues (21). Therefore, improving current understanding of miR-34a in GC is important.

The present study investigated miR-34a in clinical specimens, and in an independent, secondary cohort from The Cancer Genome Atlas (TCGA), in order to determine the prognostic value of miR-34a for patients with GC. In an attempt to unravel the properties of miR-34a, experiments were performed in GC cells. Furthermore, the downstream target of miR-34a was predicted using a bioinformatic method, and was verified in GC cells.

\section{Materials and methods}

Human tissue samples and cell lines. GC samples were obtained from 40 patients (average age, $54.5 \pm 11.73$ years; 25 males and 15 females), with clear diagnostic information, from the People's Hospital of Guangxi Zhuang Autonomous Region (Nanning, China). The patients were recruited between December 2012 and June 2014, and tissue samples were collected immediately after surgery. Two tissue samples were obtained from each patient, one sample of GC tissue, and one sample of adjacent gastric mucosa tissue. Out of the 40, a total of 20 adjacent gastric mucosa tissue samples were identified as normal gastric mucosa, whereas the other 20 samples were identified as dysplastic or metaplastic and were excluded from the present study. Overall, 10 pairs of fresh GC tissues and corresponding adjacent gastric tissues were used in the present study, due to the good quality RNA which was extracted from them. All samples were obtained following the provision of patient consent, and the experiments were approved by the Ethics Committee of the People's Hospital of Guangxi Zhuang Autonomous Region, according to the Declaration of Helsinki.

The SGC7901 GC cell line and normal gastric epithelial cells were purchased from the Shanghai Institute of Biochemistry and Cell Biology (Shanghai, China). The cells were cultured in RPMI-1640 medium (Hyclone, Logan, UT, USA) with $10 \%$ fetal bovine serum (FBS) and incubated at $37^{\circ} \mathrm{C}$ with $5 \% \mathrm{CO}_{2}$.

Immunohistochemistry (IHC). All the GC specimens were sectioned at $4 \mu \mathrm{m}$ for IHC staining, according to the manufacturer's instructions, using a DAKO REAL EnVision Detection system (Dako, Glostrup, Denmark). The slides were retrieved in boiling water for $3 \mathrm{~min}$ and cooled at room temperature. Primary rabbit anti-human polyclonal MET antibody (1:200; cat. no. sc-161; Santa Cruz Biotechnology, Inc., Dallas, TX, USA) was used to detect the expression of MET in the GC tissues. A semi-quantitative method was used to evaluate the MET staining in the GC tissues independently by two pathologists, according to the staining intensity and the percentage of positive cells.

RNA extraction and reverse transcription-quantitative polymerase chain reaction $(R T-q P C R)$. Total miRNA was extracted from the human tissue samples and the GC cells using RNAiso for Small RNA (Takara, Bio, Inc., Otsu, Japan), according to the manufacturer's instructions. A Taqman MicroRNA Reverse Transcription kit (Applied Biosystems Life Technologies, Foster City, CA, USA) was used to synthesize cDNA, and the expression of miR-34a was detected using a Taqman MicroRNA assay (Applied Biosystems Life Technologies), according to the manufacturer's instructions. U6 small nuclear RNA was used as an internal control.

Total mRNA was extracted from the cell lines and fresh GC tissue samples and was detected using a Nanodrop ND 2000 (Thermo Fisher Scientific, Inc., Wilmington, DE, USA). qPCR was performed to assess the expression of MET using an RT-PCR kit (Takara, Bio, Inc.). GAPDH was set as an internal control. All the reactions were performed at least three times using a CFX96 RT-PCR detection system (Bio-Rad Laboratories, Inc., Hercules, CA, USA). Briefly $2 \mu \mathrm{l}$ cDNA samples were subjected to PCR, using SYBR Premix Ex Taq (Takara, Bio, Inc.). The following primers were used: MET, forward GTAAGTGCCCGAAGTGTA, reverse TTTCTTGCCATCATTGTC; and GAPDH, forward TGTGGGCATCAATGGATTTGG, and reverse ACACCATGTATTCCGGGTCAAT (Invitrogen Life Technologies, Carlsbad, CA, USA). The PCR cycling conditions were as follows: $95^{\circ} \mathrm{C}$ for $30 \mathrm{sec}, 40$ cycles at $95^{\circ} \mathrm{C}$ for $5 \mathrm{sec}$ and $60^{\circ} \mathrm{C}$ for $30 \mathrm{sec}$, and final dissociation at $95^{\circ} \mathrm{C}$ for $15 \mathrm{sec}, 60^{\circ} \mathrm{C}$ for $30 \mathrm{sec}$ and $95^{\circ} \mathrm{C}$ for $15 \mathrm{sec}$. The mRNA expression levels were quantified using the $2^{-\Delta \Delta C t}$ method (22).

Transfection of miRNA reagents. The miRNA mimic and non-specific control were purchased from Guangzhou Ribobio Co., Ltd. (Guangzhou, China), which were transfected into the SGC7901 GC cells at a concentration of $100 \mathrm{nmol} / \mathrm{l}$ using Lipofectamine 2000 (Invitrogen Life Technologies), according to the manufacturer's instructions. The culture medium was replaced $6 \mathrm{~h}$ after transfection at $37^{\circ} \mathrm{C}$.

Cell proliferation assay. The SGC7901 GC cells $\left(1 \times 10^{4}\right.$ cells/well $)$ were seeded into a 96 -well plate and continuously cultured for $0,24,48$ and $72 \mathrm{~h}$ at $37^{\circ} \mathrm{C}$. At each time interval, $20 \mu \mathrm{l}$ Cell Counting Kit-8 (CCK8; Beyotime Institute of Biotechnology, Haimen, China) was added and the cells were incubated at $37^{\circ} \mathrm{C}$ for $2 \mathrm{~h}$, prior to detecting the absorbance at $450 \mathrm{~nm}$ (Multiskan; Thermo Fisher Scientific, Inc., Waltham, MA, USA).

Colony formation assay. Single SGC7901 cells (300 cells/well) were seeded into a 6 -well plate and cultured for 10 days. The colonies formed by the single SGC7901 cells were then fixed in $4 \%$ paraformaldehyde for $20 \mathrm{~min}$ and stained with $1 \%$ crystal violet for $10 \mathrm{~min}$ at room temperature. The numbers of colonies were manually counted and statistically analyzed.

Cell invasion assay. To evaluate the invasiveness of the GC cells, a Transwell invasion assay was performed. The upper surface of the chamber was pre-coated with a $10 \mu \mathrm{l}$ mixture of Matrigel (BD Biosciences, Franklin Lakes, NJ, USA) and serum free medium $(1: 1, \mathrm{v} / \mathrm{v})$. The cells $\left(1 \times 10^{4}\right.$ cells $)$ were suspended in $200 \mu \mathrm{l}$ serum-free medium and added to the upper chamber of the Transwell (EMD Millipore, Billerica, MA, USA), which 
was inserted into a 24-well plate. In the lower chamber, $500 \mu 1$ RPMI-1640 with 10\% FBS was added. Following incubation for $24 \mathrm{~h}$ at $37^{\circ} \mathrm{C}$, the cells on the upper chamber were removed and the cells on the lower chamber were fixed and stained with crystal violet. Images of the invaded cells were captured in five randomly-selected fields using a microscope (CX31; Olympus Corporation, Tokyo, Japan) and counted manually.

Bioinformatic analysis. The TCGA database (https://tcgadata.nci.nih.gov/tcga/tcgaHome2.jsp) was used to investigate the association between the expression of miR-34a and clinicopathological features, and the prognosis of patients with GC. A designed web tool (http://www.cbioportal.org/public-portal/) was used for examining the TCGA database $(23,24)$. The cut-off value for miR-34a in the GC of the TCGA cohort was set using $X$ tile software (version 3.6.1; Yale University, New Haven, CT, USA) (25). For miRNA target prediction, three popular public databases [PicTar (http://pictar.mdc-berlin. de/) (26), DIANA Tools (http://diana.imis.athena-innovation. gr/DianaTools/index.php) (27) and miRTarBase (http://mirtarbase.mbc.nctu.edu.tw/) (28)] were scanned. The selected Novoseek-inferred disease associations for MET was reviewed using the integrated gene database, GeneCards (http://www. genecards.org/).

Western blotting. Western blotting was performed to detect the protein level of MET in different samples. The GC cells were harvested $72 \mathrm{~h}$ after transfection. Total protein was extracted, according to the manufacturer's instructions of the protein extraction kit (Beyotime Institute of Biotechnology) and concentrations were determined using an Enhanced BCA Protein Assay kit (Beyotime Institute of Biotechnology). A total of $50 \mu \mathrm{g}$ protein was loaded into each lane and separated on a $15 \%$ gel, which was then wet transferred onto a polyvinylidene difluoride membrane (EMD Millipore). Following blocking with $5 \%$ milk powder, the membranes were incubated with rabbit anti-human polyclonal MET antibody (cat. no. sc-161; Santa Cruz Biotechnology, Inc.) and mouse anti-human monoclonal GAPDH antibody (cat. no. sc-322233) Santa Cruz Biotechnology, Inc.), respectively, overnight at $4^{\circ} \mathrm{C}$. Subsequently, horseradish peroxidase (HRP)-labeled secondary antibody (Beyotime Institute of Biotechnology) was added and incubated at room temperature for $2 \mathrm{~h}$. All primary antibodies were used at a concentration of 1:500 and the second antibody was used at a dilution of 1:2,000. The proteins were visualized using a Pierce ECL chemiluminescent substrates (Thermo Fisher Scientific, Inc.) and analyzed. GAPDH was used as an internal control.

Statistical analyses. All statistical analyses were performed using SPSS version 20.0 software (IBM, Armonk, NY, USA). Student's t-test was used for normally distributed data. Bonferron's-corrected Mann-Whitney U test was used to assess the expression of miR-34a in the different $\mathrm{GC}$ specimens. Correlation between the expression of miR-663 and the clinicopathological features of the patients was determined using Pearson's $\chi^{2}$ test. The prognostic value of miR-34a was estimated using a Kaplan-Meier survive curve. Data are presented as the mean \pm standard deviation. $\mathrm{P}<0.05$ $\left(^{*}\right)$ and $\mathrm{P}<0.01\left(^{* *}\right)$ were considered to indicate a statistically significant difference. All experiments were performed at least three times using triplicate samples.

\section{Results}

miR-34a is negatively correlated with invasion/metastasis and the prognosis of patients with GC. On comparing the levels of miR-34a in GC tissues and normal gastric epithelial, miR-34a was expressed at lower levels in the GC tissues,

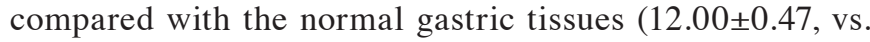
$7.06 \pm 0.33$; $\mathrm{P}<0.01$; Fig. 1A). The correlation between miR-34a and the invasion and metastasis of the GC cells was also evaluated in the GC specimens. The results indicated that miR-34a was significantly lower in cases exhibiting serosa invasion and significantly higher in cases exhibiting musocal invasion $(\mathrm{P}<0.01$; Fig. 1B). Low expression levels of miR-34a were detected in the GC specimens exhibiting lymph node metastasis (27/40; $\mathrm{P}<0.01$; Fig. 1C). These results indicated that miR-34a was negatively correlated with the depth of invasion and lymph node metastasis in the GC specimens. These results were also confirmed in an independent cohort from the TCGA database, which was used to investigate the association between miR-34a and clinicopathological features and the prognosis of patients with GC. Among the TCGA cohort $(n=352)$ with diagnostic information, 157 cases had complete follow-up information. The results demonstrated that the levels of miR-34a were significantly lower in T3 and T4, compared with T1 (P<0.05; Fig. 1D). A Kaplan-Meier survival curve was used to analyze the predictive value of miR-34a GC samples. The results indicated that patients with high expression levels of miR-34a had a significantly longer duration of survival following surgery, compared with those with low expression levels of miR-34a $(\mathrm{P}<0.05$; Fig. 1E). These results indicated that low levels of miR-34a in GC suggested frequent invasion and metastasis and predicted poorer prognosis in patients.

miR-34a suppresses the proliferation, colony formation and invasion of GC cells. GES1 is a normal gastric epithelial cell line, which was used in the present study as a normal control. The levels of miR-34a were significantly lower in the SGC7901 GC cell line, compared with its level in the GES1 line $(\mathrm{P}<0.01$; Fig. $2 \mathrm{~A})$, which confirmed the findings of the expression of miR-34a in the clinical specimens. In order to determine the effect of miR-34a on the proliferation, colony formation and invasion abilities of the GC cells, an miR-34a mimic was used. The OD450 value was significantly decreased in the group transfected with the miR-34a mimic, compared with the control group ( $\mathrm{P}<0.01$; Fig. $2 \mathrm{~B})$. A colony formation assay was used as a representative assay to assess the self-renewal ability of the cancer cells. Compared with the control, the SGC7901 transfected with the miR-34a mimic exhibited a reduced colony number $(\mathrm{P}<0.01$; Fig. $2 \mathrm{C}$ and D). A cell invasion assay was used to assess the impact of miR-34a on the invasiveness of GC. The SGC7901 cells transfected with the miR-34a mimic exhibited a reduced number of invaded cells, compared with the control $(\mathrm{P}<0.01$; Fig. 2E and F). These data demonstrated that miR-34a attenuated the proliferation, colony formation and invasion of the GC cells. 

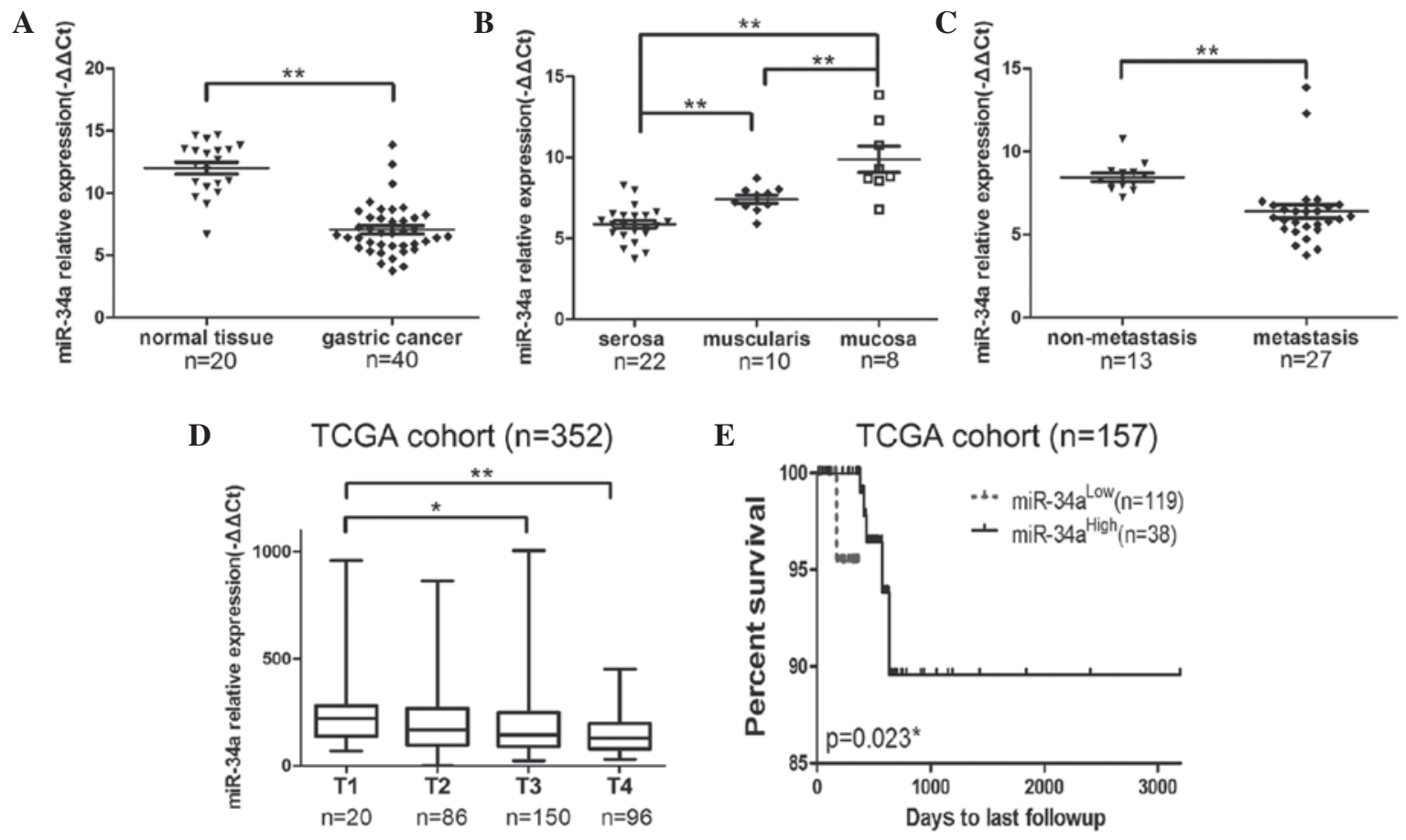

Figure 1. Clinical importance and prognostic value of miR-34a in GC. (A) Comparison between the expression of miR-34a in normal gastric tissues ( $\mathrm{n}=20$ ) and GC tissues $(\mathrm{n}=40)$. (B) Association between miR-34a and the depth of invasion in GC was evaluated. (C) Correlation between miR-34a and lymph node metastasis in GC. (D) In the TCGA GC cohort ( $\mathrm{n}=352$ ), the association between miR-34a and tumor stage (stages T1-T4) was assessed. (E) In the TCGA cohort, 157 cases had complete follow-up information. The prognostic value of miR-34a was analyzed using a Kaplan-Meier survival curve. Data are presented as the mean \pm standard deviation $\left(\right.$ "P $<0.05$ and $\left.{ }^{* *} \mathrm{P}<0.01\right)$. miR, microRNA; GC, gastric cancer; TCGA, The Cancer Genome Atlas; Ct, threshold cycle.

A

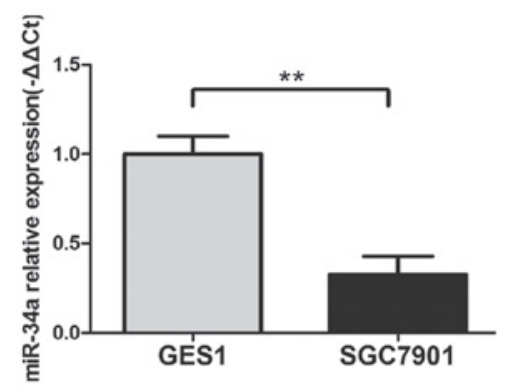

C

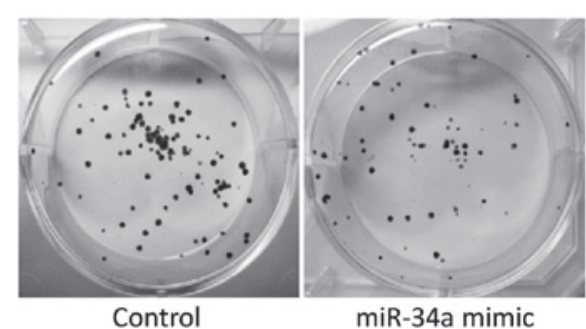

E

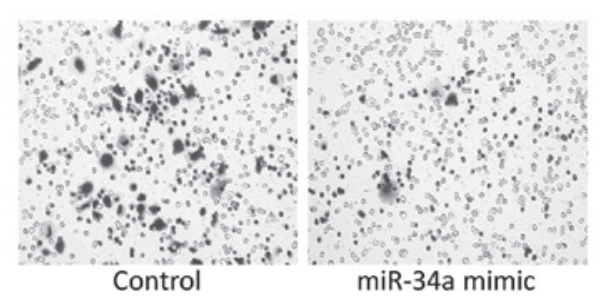

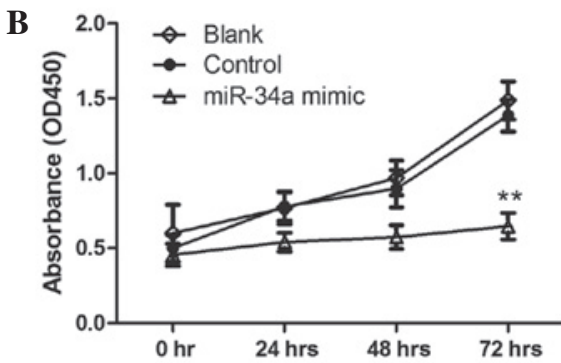

D

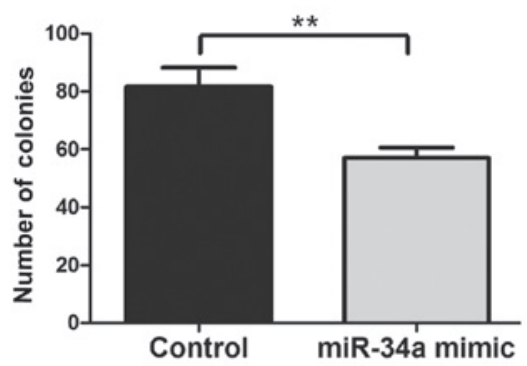

$\mathbf{F}$

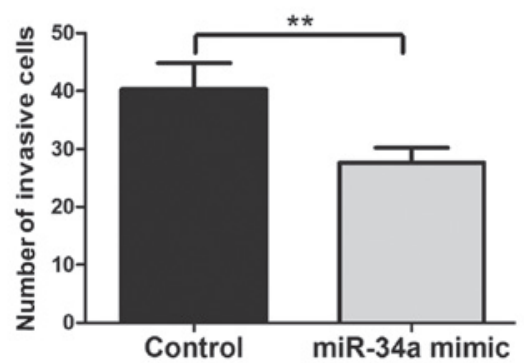

Figure 2. Effects of miR-34a on GC cell proliferation, invasion and metastasis. (A) Level of miR-34a was examined in the normal gastric epithelial cell line (GES1) and GC cell line (SGC7901). (B) A Cell Counting Kit 8 assay was used to examine the effect of miR-34a on GC cell proliferation. (C and D) A colony formation assay was used to examine the effect of miR-34a on colony formating ability in GC. (E and F) A Transwell assay was used to evaluate the effect of miR-34a on cell invasion ability in GC (magnification, $x 400$ ). Data are presented as the mean \pm standard deviation ("* $\mathrm{P}<0.01$ ). miR, microRNA; GC, gastric cancer; $\mathrm{Ct}$, threshold cycle; OD, optical density; Blank, non-specific control. 
Table I. Top 10 candidate targets of microRNA-34a, predicted using the PicTar, DIANA Tools and miRTarBase databases.

\begin{tabular}{lcc}
\hline PicTar & DIANA Tools & miRTarBase \\
\hline DLL1 & CDKN2C & BIRC3 \\
MGC34648 & ACSL4 & GRM7 \\
SYT1 & CDK4 & JAG1 \\
NAV3 & E2F1 & MYC \\
NOTCH1 & MET & MYB \\
RALGPS2 & MYB & MET \\
VAMP2 & ERLIN1 & CDK4 \\
CNTN2 & MMS19 & CCND1 \\
MET & HNF4A & BCL2 \\
ZDHHC23 & DTYMK & NOTCH1 \\
\hline
\end{tabular}

Table II. Selected Novoseek-inferred disease associations for the MET gene.

\begin{tabular}{|c|c|c|}
\hline Disease & -log (P-value) & $\begin{array}{c}\text { Number of } \\
\text { hits }\end{array}$ \\
\hline Tumor & 71.6 & 679 \\
\hline Metastasis & 69.6 & 209 \\
\hline Cancer & 65.5 & 337 \\
\hline Hereditary papillary renal cancer & 61.8 & 3 \\
\hline Gastric carcinoma & 60.2 & 66 \\
\hline Thyroid papillary carcinoma & 59.5 & 26 \\
\hline Carcinoma renal cell & 57.8 & 66 \\
\hline Carcinoma & 57.5 & 102 \\
\hline Hepatocellular carcinoma & 56.9 & 67 \\
\hline Gastric cancer & 56 & 103 \\
\hline Lymphatic metastasis & 41.1 & 1 \\
\hline Non-metastatic & 36.1 & 3 \\
\hline Metaplasia & 29.6 & 7 \\
\hline Metastatic osteosarcoma & 24.9 & 1 \\
\hline Liver metastases & 11.6 & 20 \\
\hline
\end{tabular}

miR-34a inhibits the expression of MET in GC. The present study predicted the candidate downstream targets of miR-34a in the PicTar, DIANA Tools and miRTarBase miR databases. Among the top 10 candidate genes predicted by these databases, MET was the only common gene (Fig. 3A and Table I). The predicted binding sites of MET are listed in Fig. 3B. The mRNA expression of miR-34a and MET were examined in 10 fresh GC specimens (Fig. 3C). A linear regression model was used to analyze the underlying association between the expression of miR-34a and MET. The results indicated that miR-34a was negatively correlated with MET $(\mathrm{P}<0.01 ; \mathrm{r}=-0.9526$; Fig. 3D). Following transfection with the miR-34a mimic, SGC7901 cells exhibited downregulated protein levels of MET (Fig. 3E). MET was reviewed due to its significant role in tumor and metastasis $(\mathrm{P}<0.01$; Table II).

The present study then examined MET staining in clinical specimens of GC using IHC. The results revealed high expression levels of MET in the samples with low expression of miR-34a, however, the expression level was low in the samples with high expression of miR-34a (Fig. 3F). Statistical analyses revealed that $>60 \%$ of the GC samples exhibited low miR-34a/high MET, and high miR-34a/low MET. ( $\mathrm{P}<0.01$; Fig. 3G). These results indicated that miR-34a negatively regulated $\mathrm{MET}$, which mediated the invasion and proliferation of GC.

\section{Discussion}

The dynamic expression and functions of miRNAs are closely associated with the progression of cancer and malignancy, which has been confirmed by the detection of miRNAs in clinical specimens (29). Certain miRNAs are termed 'oncomiRs' as they exhibit high levels of expression in cancer and fuel the malignant behavior of cancer cells, examples of which include miR-10b in breast cancer and miR-21 in glioblastoma $(30,31)$. Tumor suppressor miRNAs are often downregulated in cancer and inhibit the carcinogenesis and progression of cancer, for example, miR-218 has been found to attenuate the invasion, migration, proliferation and self-renewal of glioma cells via targeting Bmi (32). B-cell lymphoma (Bcl)2, an anti-apoptotic gene, has been reported to be negatively regulated by miR-15a and miR-16-1 $(33,34)$. Thus, a reduction in the expression levels of these miRNAs lead to the overexpression of $\mathrm{Bcl} 2$ and contributes to the progression of leukemia and prostate cancer. miR-34a belongs to the miR-34 family, which have been identified computationally and confirmed as evolutionarily conserved (35-38).

Human miR-34a is involved in the p53 tumor suppressor network, thus, its dysregulation is involved in cancer aggression. While miR-34a is considered to be a multifaceted miRNA in different types of cancer and has a variety of functions, a number of which appear opposite, miR-34a predominantly inhibits proliferation, progression and invasion and has been reported to be a tumor suppressor in colon cancer and glioblastoma $(17,39)$. However, miR-34a is overexpressed in hepatocellular carcinoma and is associated with liver carcinogenesis (20). A previous microRNA profile in human GC tissue revealed that miR-34a is upregulated, compared with normal gastric tissue (21). These paradoxical functions of miR-34a suggest that its effects in GC require further investigation.

Concerning the association between miR-34a and the clinicopathological features of GC, the present study examined level of miR-34a in tissue samples from patients with GC, which were confirmed in an independent TCGA cohort. The results indicated that miR-34a was a negative indicator of GC invasion/metastasis and a valuable predictor for the prognosis of patients with GC, suggesting that miR-34a may be considered as a tumor suppressor miRNA in GC.

These clinical data led to the present study investigating the role of miR-34a through a series of experiments. Consistent with the clinical data, miR-34a was expressed at a low level in the SGC7901 GC cell line and at a high level in the normal GES1 gastric epithelial cell line. Furthermore, miR-34a attenuated the proliferation, colony formation and invasion of the GC cells in vitro, which provided further evidence that miR-34a acted as a tumor suppressor miRNA in the GC cells. 
A

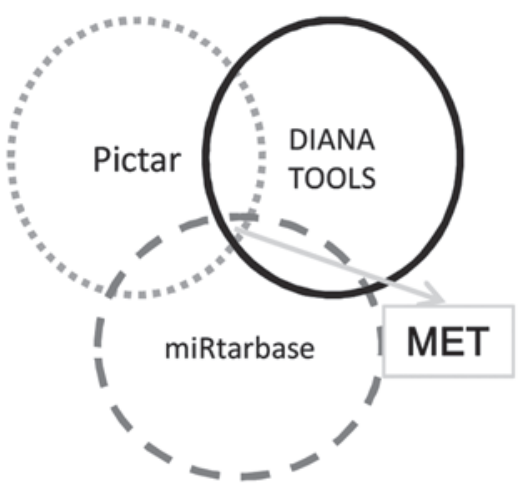

C

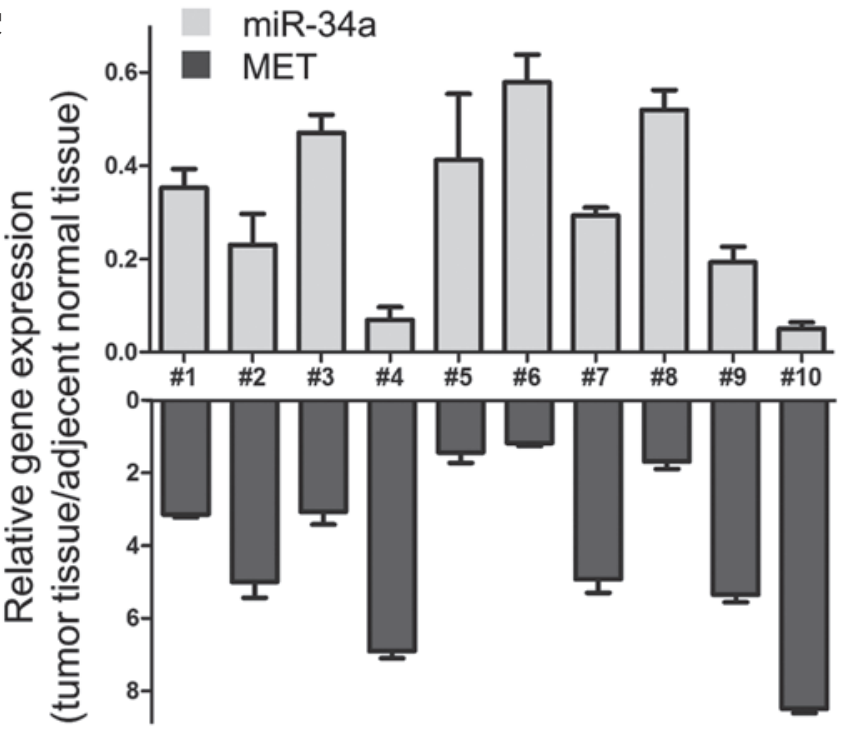

B

\begin{tabular}{|c|c|}
\hline $\begin{array}{l}\text { Position 2165-2 } \\
\text { hsa-miR-34a }\end{array}$ & $\begin{array}{c}5^{\prime} \text {...GAAUUAGAUACUUGUCACUGCCU... } \\
\text { I I I I I I I } \\
\text { 3' UGUUGGUCGAUUCUGUGACGGU }\end{array}$ \\
\hline $\begin{array}{l}\text { Position } 51-57 \text { of MET } \\
\text { hsa-miR-34a }\end{array}$ & $\begin{array}{r}5^{\prime}, \ldots \text { GUCCAAUGGUUUUUUCACUGCCU... } \\
\text { I I I I I I I } \\
\text { 3' UGUUGGUCGAUUCUGUGACGGU }\end{array}$ \\
\hline
\end{tabular}

D

Linear regression

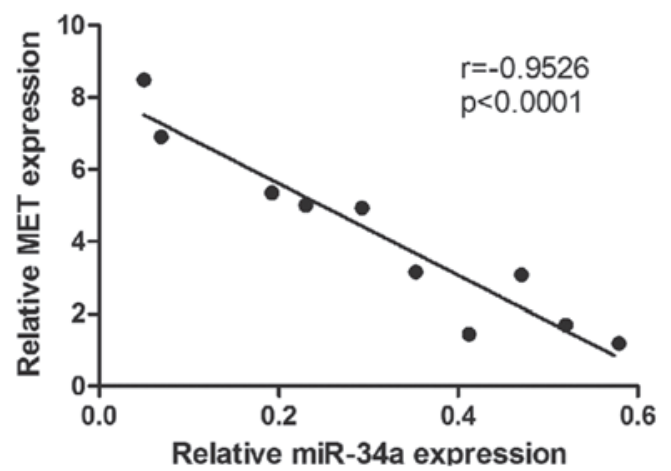

$\mathbf{E}$

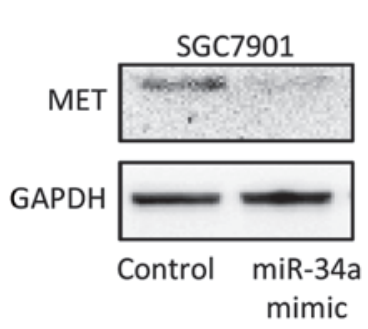

F

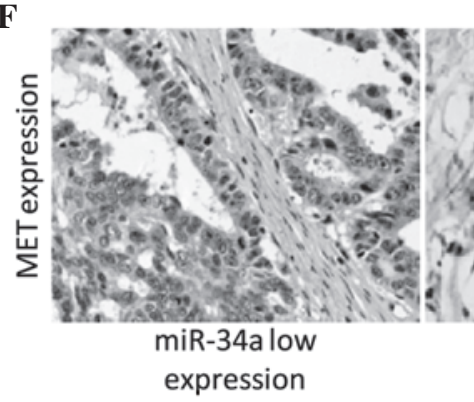

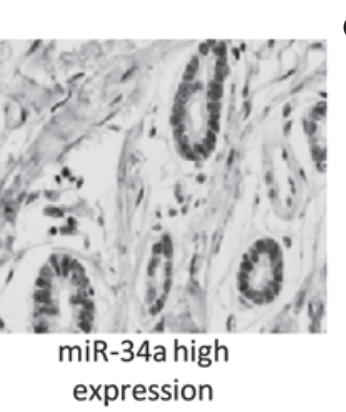

Figure 3. Molecular basis of miR-34a in modulating GC proliferation, invasion and metastasis. (A) MET was the only common target of miR-34a among the top 10 candidate genes predicted by PicTar, DIANA Tools and miRTarBase. (B) Binding site on MET of miR-34a. (C) Relative expression levels of miR-34a and MET in 10 (\#1-10) fresh GC specimens. (D) A linear regression model was used to assess the association between miR-34a and MET. (E) Regulation of MET by miR-34a was examined using western blottng. (F) MET was stained in the GC specimens in order to verify the association between miR-34a and MET (hematoxylin \& eosin; magnification, $\mathrm{x} 400$ ). (G) Percentage of consistent expression of miR-34a and MET in the GC specimens. Data are presented as the mean \pm standard deviation $\left({ }^{*} \mathrm{P}<0.05\right.$ and $\left.{ }^{* *} \mathrm{P}<0.01\right)$. miR, microRNA; GC, gastric cancer.

Since miR-34a is an miRNA, the present study bioinformatically analyzed miR-34a in the PicTar, DIANA Tools and miRTarBase miRNA targets databases. MET was found to be the only common candidate among the top 10 candidates of each database. MET was located at Chr7 q31.2, and was observed to be closely associated with tumors and metastasis. MET is considered to be an oncogene, activated in several types of cancer $(40,41)$. In colorectal cancer, the level of c-MET predicts early stage invasion and regional metastasis (42). Knockdown of c-MET in ovarian cancer significantly inhibits the extracellular signal-regulated kinase and phosphoinositide 3-kinase signaling pathways, as well as the activity of matrix metalloproteinase 2/9 (43). Co-expression of c-Met and hepatocyte growth factor in colorectal cancer allows identification of a metastatic phenotype, which correlates with advanced stage and poor survival rates (44). In hepatocellular carcinoma, low expression levels of miR-34a have been demonstrated to contribute to cancer malignancy via targeting c-Met (19). In the present study, miR-34a was found to negatively regulate the expression of MET in GC, suggesting that the tumor 
suppressing effect of miR-34a may be mediated by the downregulation of MET.

Notably, $>60 \%$ of the samples in the present study exhibited consistent expression of miR-34a and MET, with inconsistent expression observed in a minority of the GC samples. The effects of miR-34a occur in a tissue-specific manner, which involves several downstream targets. In glioma, miR-34a is reported to inhibit Notch1 and Notch2, leading to glioma growth suppression (17). In GC, survivin has been identified as another downstream target of miR-34a in modulating proliferation and invasion (18). Therefore, it is of importance to discern the interaction of miR-34a and its targets in different types of cancer.

In conclusion, with confirmation using TCGA data, the results of the present study revealed miR-34a as a tumor suppressor miRNA, which attenuated proliferation and invasion, predominantly through targeting MET in GC. These findings provide a novel perceptive to further understand the underlying molecular basis of the proliferation and invasion of $\mathrm{GC}$ and potential therapeutic approaches for GC.

\section{References}

1. Siegel R, Ma J, Zou Z and Jemal A: Cancer statistics, 2014. CA Cancer J Clin 64: 9-29, 2014

2. DeSantis CE, Lin CC, Mariotto AB, et al: Cancer treatment and survivorship statistics, 2014. CA Cancer J Clin 64: 252-271, 2014

3. Khalighinejad N, Hariri H, Behnamfar O, Yousefi A and Momeni A: Adenoviral gene therapy in gastric cancer: A review. World J Gastroenterol 14: 180-184, 2008.

4. Leja M, Wex T and Malfertheiner P: Markers for gastric cancer premalignant lesions: Where do we go? Dig Dis 30: 268-276, 2012.

5. Amedei A, Della Bella C, Silvestri E, Prisco D and D'Elios MM: T cells in gastric cancer: Friends or foes. Clin Dev Immunol 2012: 690571, 2012.

6. Toiyama Y, Okugawa Y and Goel A: Dna methylation and microRNA biomarkers for noninvasive detection of gastric and colorectal cancer. Biochem Biophys Res Commun 455: 43-57, 2014

7. Felipe AV, Oliveira J, Chang PY, et al: RNA interference: a promising therapy for gastric cancer. Asian Pac J Cancer Prev 15 5509-5515, 2014.

8. Pasquinelli AE: MicroRNAs and their targets: Recognition, regulation and an emerging reciprocal relationship. Nat Rev Genet 13: 271-282, 2012.

9. Wang Y and Lee CG: MicroRNA and cancer-focus on apoptosis. J Cell Mol Med 13: 12-23, 2009.

10. Bueno MJ, Pérez de Castro I and Malumbres M: Control of cell proliferation pathways by microRNAs. Cell Cycle 7: 3143-3148, 2008.

11. Liang LH and He XH: Macro-management of microRNAs in cell cycle progression of tumor cells and its implications in anti-cancer therapy. Acta Pharmacol Sin 32: 1311-1320, 2011

12. Lowery AJ, Miller N, McNeill RE and Kerin MJ: MicroRNAs as prognostic indicators and therapeutic targets: Potential effect on breast cancer management. Clin Cancer Res 14: 360-365, 2008.

13. Nouraee $\mathrm{N}$ and Calin GA: MicroRNAs as cancer biomarkers. Microrna 2: 102-117, 2013

14. Berindan-Neagoe I, Monroig Pdel C, Pasculli B and Calin GA: MicroRNAome genome: A treasure for cancer diagnosis and therapy. CA Cancer J Clin 64: 311-336, 2014.

15. Maroof H, Salajegheh A, Smith RA and Lam AK: Role of microRNA-34 family in cancer with particular reference to cancer angiogenesis. Exp Mol Pathol 97: 298-304, 2014

16. Balca-Silva J, Sousa Neves S, Goncalves AC, et al: Effect of miR-34b overexpression on the radiosensitivity of non-small cell lung cancer cell lines. Anticancer Res 32: 1603-1609, 2012.

17. Li Y, Guessous F, Zhang Y, et al: MicroRNA-34a inhibits glioblastoma growth by targeting multiple oncogenes. Cancer Res 69: 7569-7576, 2009.

18. Cao W, Fan R, Wang L, et al: Expression and regulatory function of miRNA-34a in targeting survivin in gastric cancer cells. Tumour Biol 34: 963-971, 2013.
19. Dang Y, Luo D, Rong M and Chen G: Underexpression of miR-34a in hepatocellular carcinoma and its contribution towards enhancement of proliferating inhibitory effects of agents targeting c-MET. PLoS One 8: e61054, 2013.

20. Pineau P, Volinia S, McJunkin K, et al: miR-221 overexpression contributes to liver tumorigenesis. Proc Natl Acad Sci USA 107: 264-269, 2010.

21. Yao Y, Suo AL, Li ZF, et al: MicroRNA profiling of human gastric cancer. Mol Med Rep 2: 963-970, 2009.

22. Braun CJ, Zhang X, Savelyeva I, Wolff S, Moll UM, Schepeler T, Ørntoft TF, Andersen CL and Dobbelstein M: p53-Responsive micrornas 192 and 215 are capable of inducing cell cycle arrest. Cancer Res 68: 10094-10104, 2008.

23. Cerami E, Gao J, Dogrusoz U, et al: The cBio cancer genomics portal: An open platform for exploring multidimensional cancer genomics data. Cancer Discov 2: 401-404, 2012.

24. Gao J, Aksoy BA, Dogrusoz U, et al: Integrative analysis of complex cancer genomics and clinical profiles using the cBioPortal. Sci Signal 6: pl1, 2013.

25. Camp RL, Dolled-Filhart M and Rimm DL: X-tile: a new bio-informatics tool for biomarker assessment and outcome-based cut-point optimization. Clin Cancer Res 10: 7252-7259, 2004.

26. Krek A, Grün D, Poy MN, et al: Combinatorial microRNA target predictions. Nat Genet 37: 495-500, 2005.

27. Zhang L, Huang J, Yang N, et al: microRNAs exhibit high frequency genomic alterations in human cancer. Proc Natl Acad Sci USA 13: 9136-9141, 2006.

28. Hsu SD, Lin FM, Wu WY, et al: miRTarBase: A database curates experimentally validated microRNA-target interactions. Nucleic Acids Res 39: D163-D169, 2011.

29. Calin GA, Ferracin M, Cimmino A, et al: A MicroRNA signature associated with prognosis and progression in chronic lymphocytic leukemia. N Engl J Med 353: 1793-1801, 2005.

30. Parrella P, Barbano R, Pasculli B, et al: Evaluation of microRNA-10b prognostic significance in a prospective cohort of breast cancer patients. Mol Cancer 13: 142, 2014.

31. Hermansen SK, Dahlrot RH, Nielsen BS, Hansen S and Kristensen BW: MiR-21 expression in the tumor cell compartment holds unfavorable prognostic value in gliomas. J Neurooncol 111: 71-81, 2013

32. Tu Y, Gao X, Li G, et al: MicroRNA-218 inhibits glioma invasion, migration, proliferation and cancer stem-like cell self-renewal by targeting the polycomb group gene Bmil. Cancer Res 73: 6046-6055, 2013.

33. Aqeilan RI, Calin GA and Croce CM: miR-15a and miR-16-1 in cancer: Discovery, function and future perspectives. Cell Death Differ 17: 215-220, 2010.

34. Bonci D, Coppola V, Musumeci M, et al: The miR-15a-miR-16-1 cluster controls prostate cancer by targeting multiple oncogenic activities. Nat Med 14: 1271-1277, 2008.

35. Houbaviy HB, Murray MF and Sharp PA: Embryonic stem cell-specific MicroRNAs. Dev Cell 5: 351-358, 2003.

36. Dostie J, Mourelatos Z, Yang M, Sharma A and Dreyfuss G: Numerous microRNPs in neuronal cells containing novel microRNAs. RNA 9: 180-186, 2003.

37. Lim LP, Glasner ME, Yekta S, Burge CB and Bartel DP: Vertebrate microRNA genes. Science 299: 1540, 2003.

38. He L, He X, Lim LP, et al: A microRNA component of the p53 tumour suppressor network. Nature 447: 1130-1134, 2007.

39. Cho WC: OncomiRs: The discovery and progress of microRNAs in cancers. Mol Cancer 6: 60, 2007.

40. Lutterbach B, Zeng Q, Davis LJ, et al: Lung cancer cell lines harboring MET gene amplification are dependent on Met for growth and survival. Cancer Res 67: 2081-2088, 2007.

41. Engelman JA, Zejnullahu K, Mitsudomi T, et al: MET amplification leads to gefitinib resistance in lung cancer by activating ERBB3 signaling. Science 316: 1039-1043, 2007.

42. Takeuchi H, Bilchik A, Saha S, et al: c-MET expression level in primary colon cancer: A predictor of tumor invasion and lymph node metastases. Clin Cancer Res 9: 1480-1488, 2003.

43. Sawada K, Radjabi AR, Shinomiya N, et al: c-Met overexpression is a prognostic factor in ovarian cancer and an effective target for inhibition of peritoneal dissemination and invasion. Cancer Res 67: 1670-1679, 2007.

44. Kammula US, Kuntz EJ, Francone TD, et al: Molecular co-expression of the c-Met oncogene and hepatocyte growth factor in primary colon cancer predicts tumor stage and clinical outcome. Cancer Lett 248: 219-228, 2007. 